\title{
Geovisualization of Sub-surface Pipelines: A 3D Approach
}

\author{
Abdul-Lateef Balogun (Corresponding author) \\ PhD Student, Department of Civil Engineering, Universiti Teknologi Petronas \\ Bandar Seri Iskandar, 31750 Tronoh, Perak Darul Ridzuan, Malaysia \\ Tel: 60-13-361-9767 E-mail: geospatial63@gmail.com \\ Abdul-Nasir Matori \\ Associate Professor, Department of Civil Engineering, Universiti Teknologi Petronas \\ Bandar Seri Iskandar, 31750 Tronoh, Perak Darul Ridzuan, Malaysia \\ Tel: 60-12-437-1617Ｅ-mail: nasrat@petronas.com.my \\ Dano Umar Lawal \\ PhD Student, Department of Civil Engineering, Universiti Teknologi Petronas \\ Bandar Seri Iskandar, 31750 Tronoh, Perak Darul Ridzuan, Malaysia \\ Tel: 60-17-736-6200Ｅ-mail: bblawaldano@gmail.com
}

Received: March 23, 2011

Accepted: June 17, 2011

doi:10.5539/mas.v5n4p158

\begin{abstract}
This century has continued to witness an ever increasing reliance on Geographical Information Systems (GIS) technology for the management of utilities' pipelines world over. Underground cables and pipelines are required to transport essential utilities such as oil, gas, water and electricity from one part of the city to another. Unlike on-surface pipelines, the fact that subsurface pipelines are hidden from the naked eyes makes them susceptible to neglect and damages without being easily noticed. Such damages and consequent pipe failures often have disastrous consequences on the environment and its inhabitants. A common source of subsurface pipeline damage is the accidental cutting of pipelines by excavation workers, oblivious of the precise underground location of such pipelines. This is largely due to the fact that pertinent decisions are usually taken using two dimensional (2D) maps as reference; however, information contained in 2D maps are often misinterpreted by both field workers and professionals alike.

Three dimensional (3D) maps are increasingly becoming popular due to their ability to overcome the limitations inherent in (2D) maps. They also aid the proper conceptualization of subsurface pipelines thereby making it easier to work around these pipelines without endangering them. One major drawback though is the exorbitant cost of most of the GIS packages that support the 3D modelling and visualization of subsurface pipelines. Furthermore, the advanced languages used in building many of these packages make it difficult for non-GIS experts and professionals to relate with them. Since people from diverse disciplines (without strong GIS background) need to visualize and analyze these subsurface pipelines on a regular basis, it is pertinent to develop a system capable of performing basic 3D visualization functions, in addition to being user-friendly and highly affordable. This paper discusses such a technique, utilizing open-source software.
\end{abstract}

Keywords: 3D, GIS, Pipeline, Geovisualization, Modelling

\section{Introduction}

Proper visualization of pipelines such as oil and gasoline pipelines, water supply, sewage, power supply, heat supply, industrial pipelines e.t.c. is a top-level challenge for the geospatial industry. Due to the essential functions performed by the pipelines that transport these essential utilities, most governments are committed to ensuring their safety. Similarly, most governments are keen on ensuring that they are properly managed because of the important functions performed by these pipelines. In order to monitor these underground pipes, as well as predict the extent to which the construction of new utilities and structures will affect them, it is essential to be able to visualize them properly. An integral part of a pipeline management system is the availability of documents (maps) that offer related graphic information about existing pipelines. These maps serve as visualization tools needed to monitor the underground infrastructures. However, the visualization of utility networks has usually been on two dimensional (2D) maps, and also the information therein is often misinterpreted particularly when made available to third parties like unskilled labourers, or non-professionals (AbdulRahman et al, 2006).Such misinterpretations have led to severe accidents which consist of blind-cutting off of water, gas, heat supply and so on (Du, 2006). Other equally serious accidents such as the cut-off of communication lines and even the overflow of sewage had been caused by blind-cutting or fault damaging. To be able to resolve these perennial problems, three dimensional (3D) visualization of utilities is most essential (AbdulRahman, 2004; Du, 2005; Chong, 2006). 


\section{Objective}

The primary objective of this work is to device a Geographical Information System (GIS) model for visualizing sub-surface pipelines in full, three dimensional (3D) formats. This model will be an improvement on current two-dimensional (2D) visualization techniques and will be a departure from existing 3D visualization models in terms of huge operational and procurement cost.

\section{Literature Review}

The definition of 3D GIS is similar to that of 2D GIS, and the fundamental difference between them is the fact that the information in the former is associated with three-dimensional spatial phenomena (Abdul-Rahman, Zlatanova and Pilouk, 2001). Hence, it is expected that 3D GIS should be able to perform the same tasks as 2D GIS.

2D Geographic Information Systems (GIS) are designed to handle information relating to spatial locations. It is a collection of computer hardware, software, geographic data, people, and organizations, for collecting, storing, analyzing and disseminating all types of geographically referenced information (Dueker, 1989). The most common understanding of a GIS emphasizes it as a tool for storing, retrieving, transforming and displaying spatial data (Burrough, 1986). A powerful decision making tool, GIS is increasingly being relied upon by a wide range of professionals from various fields for the purpose of accessing, viewing, relating and analyzing maps and data. When combined with other applications, GIS has the unprecedented capability to manage, aggregate, quality-control, preserve, and secure data. Since early '90s, GIS has become a sophisticated system for maintaining and analyzing spatial and thematic information on spatial objects, and the need for 3D information is rapidly increasing especially due to the limitations of 2D GIS in analyzing situations such as water flood models, geological models, Air pollution models (Van wees, 2002).

While Computer Aided Design (CAD) systems could be said to have a long history in managing information in three dimensions, the same cannot be said for the development of GIS which tends to focus more on database management. It has since been realized that an integration of CAD systems and GIS would be beneficial to both parties. Having discovered their seeming inter-dependence on each other, the development of CAD systems and GIS is today moving the two systems closer to each other. For instance, while CAD is developed with the ability to work with 2D projections, define complex hierarchy of attribute, as well as perform GIS-like analysis, the GIS community on its own part demands more realistic 3D visualization, 3D editing capabilities, and better navigation possibilities ( $\mathrm{Pu}$ and Zlatanova, 2006). Figure 1 provides a graphical description of the integration of GIS and CADD.

In spite of apparent limitations, the DBMS-CAD integration has indeed brought tremendous benefits in the area of visualization and data editing. Having 3D data stored in a database, the user has the possibility to extract only a limited set of data, thereby significantly reducing the time required for loading. Similarly, locating, editing and examining a particular object also becomes easier, faster, and more convenient (Rahman et al, 2002).

While other disciplines such as urban planning, environmental monitoring, telecommunications, public rescue operations, landscape planning etc have met the need for 3D geoinformation (Stoter and Zlatanova, 2003), the same cannot necessarily be said for pipeline management ; most of the existing pipelines today are viewed and managed in 2D (Hu Zhuowei, 2005).

2D GIS visualization only has the ability to map objects to 2D plane and represent them with symbols. With this method, it is impossible to know the 3D topological relationship among pipelines (Hu Zhuowei et al, 2005). Similarly, the map distance in 2D is inaccurate because it is significantly different from the real distance in $3 \mathrm{D}$ that is measured linearly along the pipe axis (Pubellier, 2003). Consequently, the information contained in 2D maps is often misinterpreted particularly when made available to third parties like unskilled labourers, or non-professionals (AbdulRahman et al, 2006). Such misinterpretations have led to severe accidents which consist of blind-cutting off of water, gas, heat supply and so on (Du, 2006). Other equally serious accidents such as the cut-off of communication lines and even the overflow of sewage had been caused by blind-cutting or fault damaging. To be able to resolve these perennial problems, three dimensional (3D) visualization of utilities is most essential (AbdulRahman, 2004; Du, 2005; Chong, 2006).

There are numerous examples of construction projects that led to disastrous and extremely expensive utility infrastructure repairs due to the non-availability of an extensive and accurate utility mapping information, which a 3D map would have provided. (Morgan et al, 1996). Accurate mapping of the infrastructure will help in maintaining proper record of the pipelines, and help in finding out accurately the defects developed over time. The final layout developed using GIS could be called a thematic map which will become the source for monitoring and locating the pipes (Yelakanti et al, 2003). The integration of GIS with infrastructure management Information system (MIS) offers a new and innovative technique of creating smart maps which enhance better communication of information. Since GIS is moving towards the 3D environment at a very fast pace, it is pertinent to provide the necessary graphical and information handling technology to record information on all buried utilities in user-friendly, three dimensional underground maps. 


\section{3D Visualization}

The definition of 3D GIS is comparable to that of 2D GIS, and the basic variation between them is the fact that the data in the former is related to three-dimensional spatial phenomena (Abdul-Rahman, Zlatanova and Pilouk, 2001). Therefore, it's anticipated that 3D GIS ought to have the ability to execute the same tasks as 2D GIS. Since the entire world all around us is three-dimensional (3D), it is only normal that presentations of GIS information need to move in this direction also. Similarly, the growing need for 3D information along with the remarkable developments recorded in 3D data collection methods have aided the progress of 3D GIS (Stoter and Zlatanova, 2003). Although CAD programs could be stated to have an extended historical past in managing information in three dimensions, with their style focused on building great 3D editing resources and efficient visualization, the same can't be stated for the advancement of GIS which tends to emphasise a lot more on database management. It has since been recognized that an integration of CAD programs and GIS will be advantageous to the two parties. Having found their seeming inter-dependence on one another, the growth of CAD systems and GIS are these days shifting the two systems nearer to one another. For example, while CAD is created with the capability to function with 2D projections, outline complicated hierarchy of attribute, and also carry out GIS-like analysis, the GIS community on its own part demands much more practical 3D visualization , 3D editing capabilities, and greater navigation prospects (Pu and Zlatanova, 2006).

\section{Modelling pipelines}

Any time a utility provider digs a hole in the road there's a threat of damage to another utility's buried products. This is in addition to the effect on the nearby surroundings and traffic disruption. Locating underground assets is believed by some working in the industry to be a 'rather hit and miss affair'; this is due to the significant quantity of 'dry holes' (i.e. excavations that seek out, but fall short, to locate buried utilities) which are dug each year.(Costello, S.B. et al, 2007).In recent times, the location of underground utilities has been based on data that locates the utility line in only two dimensions, normally in the form of a line on a map with occasional local indications of depth. Unfortunately, the information gotten from such maps are often incomplete and inaccurate. The third dimension, i.e. depth, is essential to the development of correct three-dimensional designs of all buried utilities, which in turn are a requirement for the installation or on-line replacement of utilities by present day trenchless methods (Costello, S.B. et al, 2007).Hence, the need for 3D modelling and visualization of these utilities.

Five criterions have been identified as essential factors which ought to be present in-order to have detailed, reliable models of real world objects like pipelines (El-Hakim, 2008). These include the following:

1. The resolution of the rendered model should ideally match what is perceptible by the human eyes on a real visit or physical interaction.

2. For the acquired data, accuracy and noise level must be sufficiently high, otherwise all subsequent processing on this data will be negatively affected. It also has to guarantee that the accuracy of the final model matches the application specifications.

3. Data collection and modelling tools should be highly automated in all operations in order to complete a given project in a reasonable time.

4. Generate efficient sized models, without loss of desired details, so they can be viewed interactively by the application software and hardware.

5. Overall low cost.

Unfortunately, there is no single system with the capability to meet all these requirements for all 3D visualization cases. Modern technology imposes a compromise given that it's generally unlikely, for instance, to acquire data

with resolution matching the smallest detail on a surface within a reasonable time, or that we are able to accomplish substantial precision and resolution with complete automation at low price all at once. Consequently, the aim of several research pursuits is to seek out remedies to these recurring concerns. This paper zeroes in on the last concern identified by (El-Hakim, 2008) i.e. adopting a modelling cum visualization technique with an overall low cost.

\section{An Ensemble approach to 3D Modelling and Visualization}

The research procedure was divided into stages and these stages were followed in a sequential manner in-order to achieve the laid down objectives.

1. Phase 1

- Data collection

2. Phase 2

- Data processing

3. Phase 3

- Result \& Analysis

- Visualization 
In this paper, emphasis will be on the third phase i.e. visualization of the pipelines in 3D format. Three different softwares namely: ArcGIS 9.2 (3D Analyst), VRML plug-ins and 3DS Max will be used for this purpose. Viewing the datasets i.e. pipelines directly using ArcMap, the pipelines are represented in 2D format as shown in figure 2 .

It can be seen that the pipelines (purple), land lots (blue) and other features are all represented using either points, lines or polygons. This doesn't give a very clear picture of the features. Hence, the need to adopt other functionalities. After extruding in ArcGIS, the format of the point features was turned into vertical lines while the lines were turned into walls as seen in figure 3.

In spite of being able to give an improved display in comparison to 2D data format, using ArcScene for visualizing the pipelines nonetheless has limitations since the pipelines are not able to be represented in true 3D cylindrical formats. Instead, they are being displayed as extruded wall surfaces. To overcome this limitation, it is necessary to transform the features into other 3D formats. Benefiting from enhanced computer software interoperability, Virtual Reality Modeling Language (VRML) plug-ins and Autodesk 3D studio Max have been utilized to generate a 3D environment. For this task, the Cortona3D Viewer, which functions as a VRML plug-in was made use of to render the features through the process of importing them in from ArcScene. The VRML viewer is shown in figure 4.

After exporting to the VRML format, the dataset was further exported into 3DS format utilizing the Autodesk 3D Studio Max application. Although Autodesk 3D studio Max is unable to exhibit pipes in cylindrical format, it nonetheless supports the 3D modelling software program, Google sketch-up; this makes clear the reason why the data was converted to this format before getting exported to the Sketch-up software. Sketch-up does not possess the capability to directly read or interpret ArcGIS or VRML file formats, however it can read 3DS files from Autodesk 3D Studio Max. Therefore, the conversion from .mdb to .wrl, and from .wrl to 3DS.

\section{Results}

Using the preliminary datasets available, the pipelines can actually be viewed underneath the building lots, in 3D form as shown in figure 5. It can be seen that the $3 \mathrm{D}$ view is definitely clearer and better than the $2 \mathrm{D}$ format shown in figure 6.

The cylindrical pipelines and other features (buildings) can be viewed in three dimensional formats as shown in figure 7 .

Whilst the pipelines' attributes are still retained and remain intact within the geodatabase from where a variety of queries could be executed in-order to retrieve pertinent information regarding the pipelines together with other features in real-time, the modelling and visualization in 3D format enhances the display and perception of the pipes and helps make it simpler to relate with it, likewise comprehend its workings. In a similar fashion, the methodology proposed in this paper is simpler to adopt in addition to being a lot more cost-effective than current 3D GIS.

\section{Discussion}

There is an increasing need to visualize underground pipelines in 3D format, but there are few packages in the market with the ability to perfectly visualize and analyze GIS data in 3D format. The available products with this functionality are often expensive and beyond the reach of most GIS users. This research has shown that pipelines can actually be visualized in a 3 dimensional format, but not as a complete solution to all the problems involved in representing $3 \mathrm{D}$ features. The major advantage of such 3D visualization is that it gives a clearer picture of the pipelines than representing them in 2D. However, the major disadvantage is that there are no abilities to represent the pipes as cylinders within the ESRI environment and this necessitates the use of stand-alone software like Sketch-up for the visualization, while analysis is still performed in the ESRI environment.

$3 \mathrm{D}$ visualization is very useful in the planning phase of a project because it gives a very clear picture of what the pipes underground look like. Excavation and other workers, and other stake holders will understand the pipes better in this format. The approach presented in this paper is quite effective for this purpose and equally affordable.

\section{Conclusion and Further Research}

Comparing these results with results from a similar study conducted in Pahang, Malaysia, it will be seen that the methodology adopted seems quite effective in dsiplaying the underground pipelines in 3D format.However, to further validate this approach, it is necessary to use a larger dataset covering a wide and diverse types of pipelines.Realistically, there are different types of pipes and cables underneath the ground. Water pipes, oil pipes, gas pipes, electrical cables etc. While this paper has focused only on a particular type of pipeline, ongoing research aims to see how other types of pipes and cables that exist simultaneously beneath the earth's surface can be visualized together concurrently, in 3D format. Such user-friendly and affordable top-level 3D visualization technique will go a long way in ensuring that these pipes are not accidentally cut-off or tampered with by workers during construction or other projects that require excavation works.

Furthermore, in continuation of our ongoing research, efforts will be made to integrate an enhanced and affordable 3D visualization technique with a decision support system in-order to determine and subsequently 
visualize the optimal pipeline route in a given community. By adopting an effective 3D visualization technique in displaying the pipeline routes, all stakeholders in the route selection process will be provided with clear, unambiguous views of the route and this will make it possible to reach a consensus on the best pipeline route possible.

\section{References}

AbdulRahman, A, Zlatanova, S, and Pilouk, M. (2002). Trends in 3D GIS development. Journal of Geospatial Engineering. The Hong Kong Institution of Engineering Surveyors.

Abdul-Rahman, A., Zlatanova, S., Coors, V. (2006). Innovations in 3D geoinformation Systems. SpringerLink.

Abdul-Rahman, A., Held, G., Zlatanova, S. (2004). Web 3D GIS for Urban environments. GDMC Publications, Netherlands.

Bernardini, F. \& Rushmeier, H. (2002). The 3D model acquisition pipeline. Computer Graphics Forum 21(2): 149-172.

Burrough, P.A. (1986). Principles of Geographic Information Systems for Land and Resources Assessment. Monographs on soil and resources survey, the Oxford University Press.

Chong Sc. (2006). Registration of way leave (Cable and Pipeline) into the Dutch Cadastre. GDMC Publications. Delft University of Technology.

Costello, S.B et al. (2007). Underground asset location and condition assessment technologies. Tunneling and underground space technology. Science Direct.

Du,Y. (2005). 3D Visualization of Urban Pipelines. Case study Report. GDMC Publications.

Du, Y., Zlatanova, S. (2006). An approach for 3D visualization of pipelines. Springer Berlin Heidelberg.

Duddeck, H. (1996). Challenges to tunneling Engineering. Tunneling and underground Space Technology 11. Technical University of Braunschweig, Germany.

Dueker, K. J., Kjcrne, D. (1989). Multipurpose cadastre: Terms and Definitions. Falls Church. VA:ASPRS and ACSM.

El-Hakim, S. (2008). 3-D Data modelling and Visualization. ISPRS Congress Book.

Morgan, A., Taylor, N. (1996). The technical and economic case for the use of three dimensional mapping for the installation of electric power cables. Proceedings of the International No-Dig '96 New Orleans, Louisiana, USA

$\mathrm{Pu}, \mathrm{S}$., Zlatanova, S. (2006). Integration of GIS and CAD at DBMS Level. UDMS.

Wees, J.D. Van, R.W. Versseput, H.J. Simmerlink, R.R.L. Allard and H.J.M. Pagnier. (2002). Netherlands Institute of Applied Geoscience TNO- National Geological Survey, Geo-informatiedag, Ede, The Netherlands

Stoter, J and Zlatanova, S. (2003). 3D GIS, Where are we standing? Delft University of Technology, the Netherlands.

Yelakanti, V et al. (2004). Role of GIS in Pipeline industry. American Society of civil Engineers.

Zhuowei, H., Huili, G., Wenji, Z. (2005). Research of the display question in the 3D pipeline management information system. Geoscience and Remote Sensing Symposium. IEEE International.

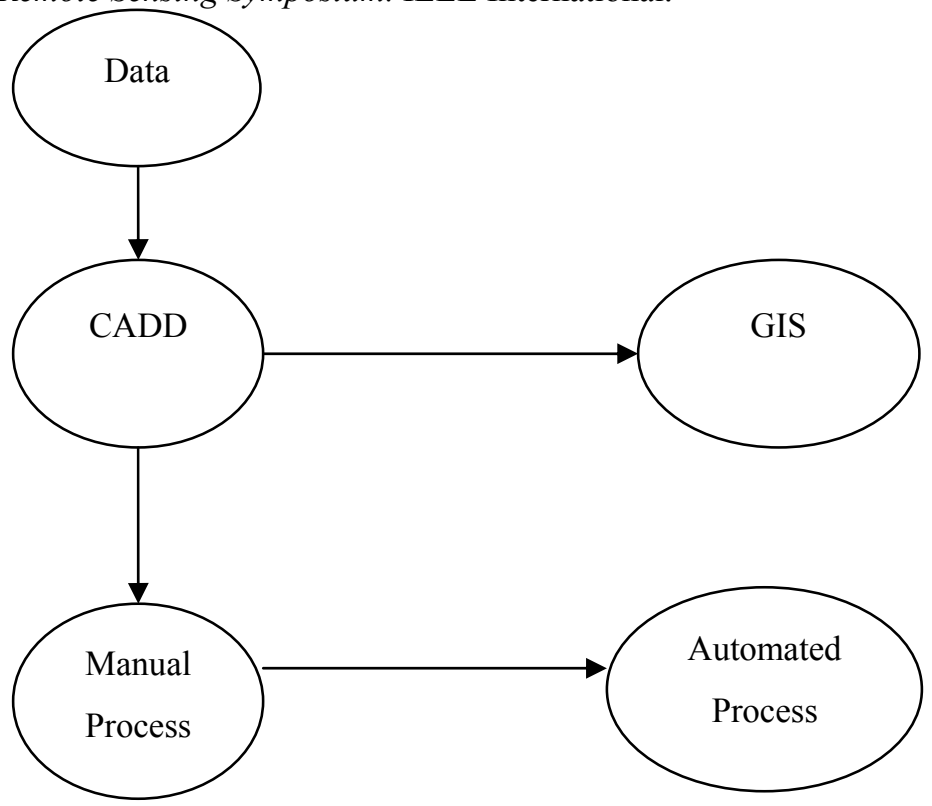

Figure 1. The Integration of GIS and CADD (After Yelakanti et al, 2003) 


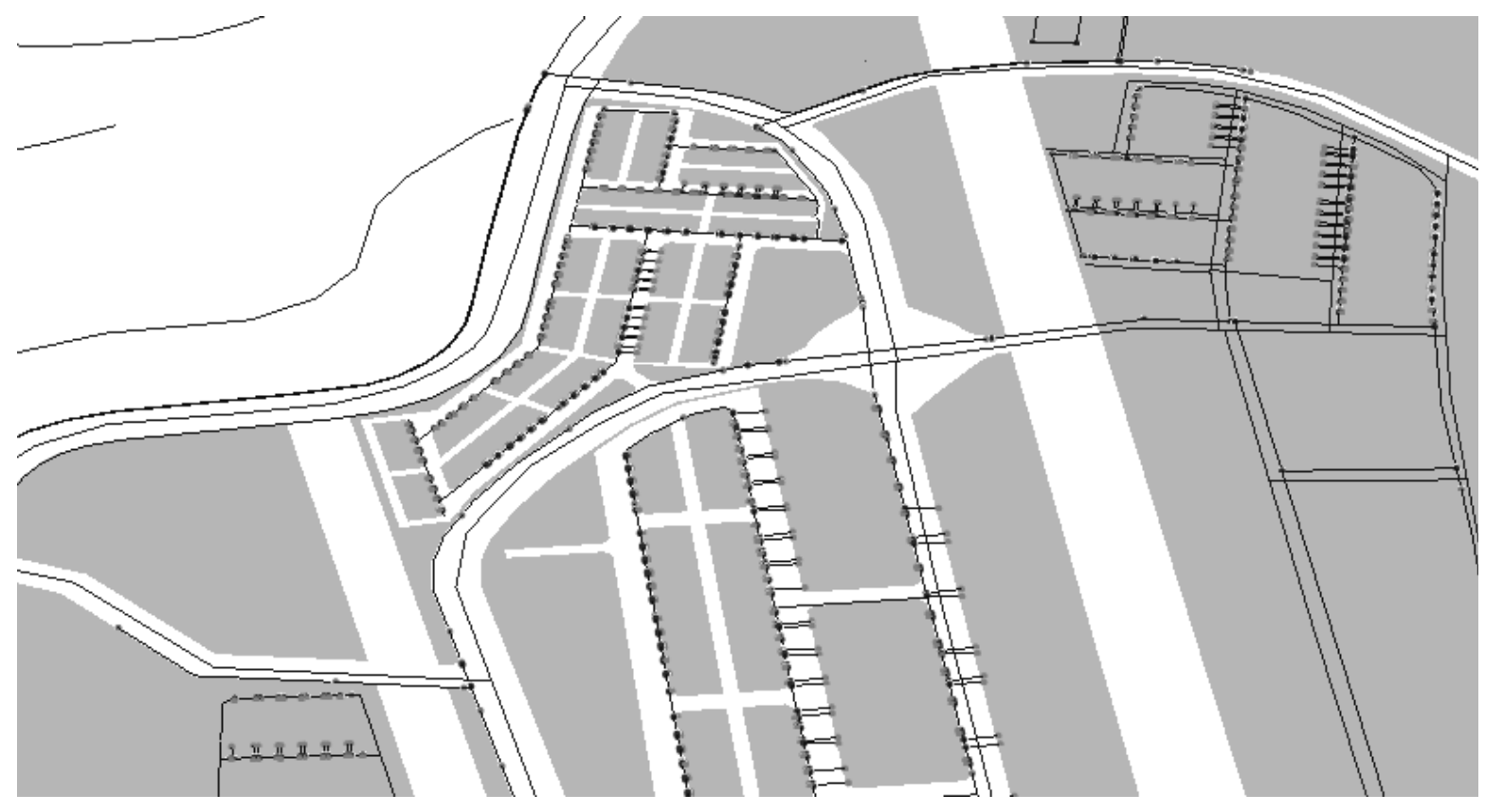

Figure 2. visualization of pipelines

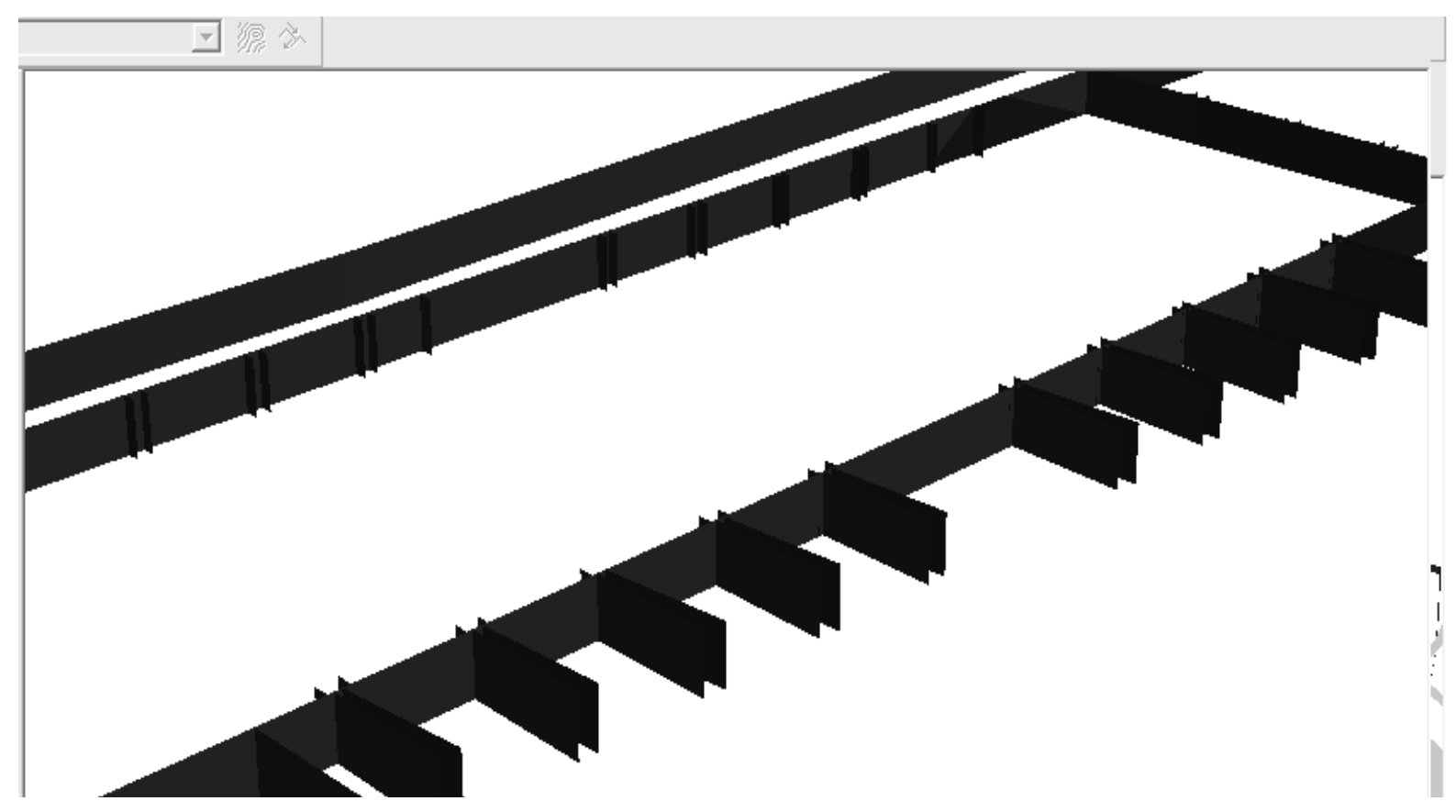

Figure 3. The pipelines are displayed as walls in ArcScene 


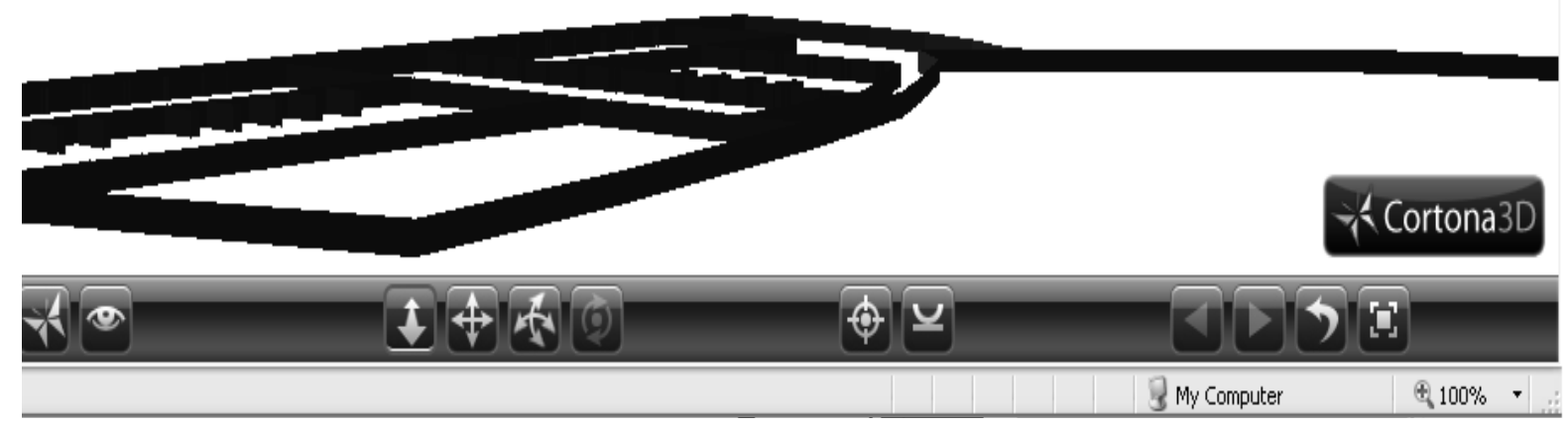

Figure 4. Pipelines being viewed via a VRML viewer (Cartona 3D)

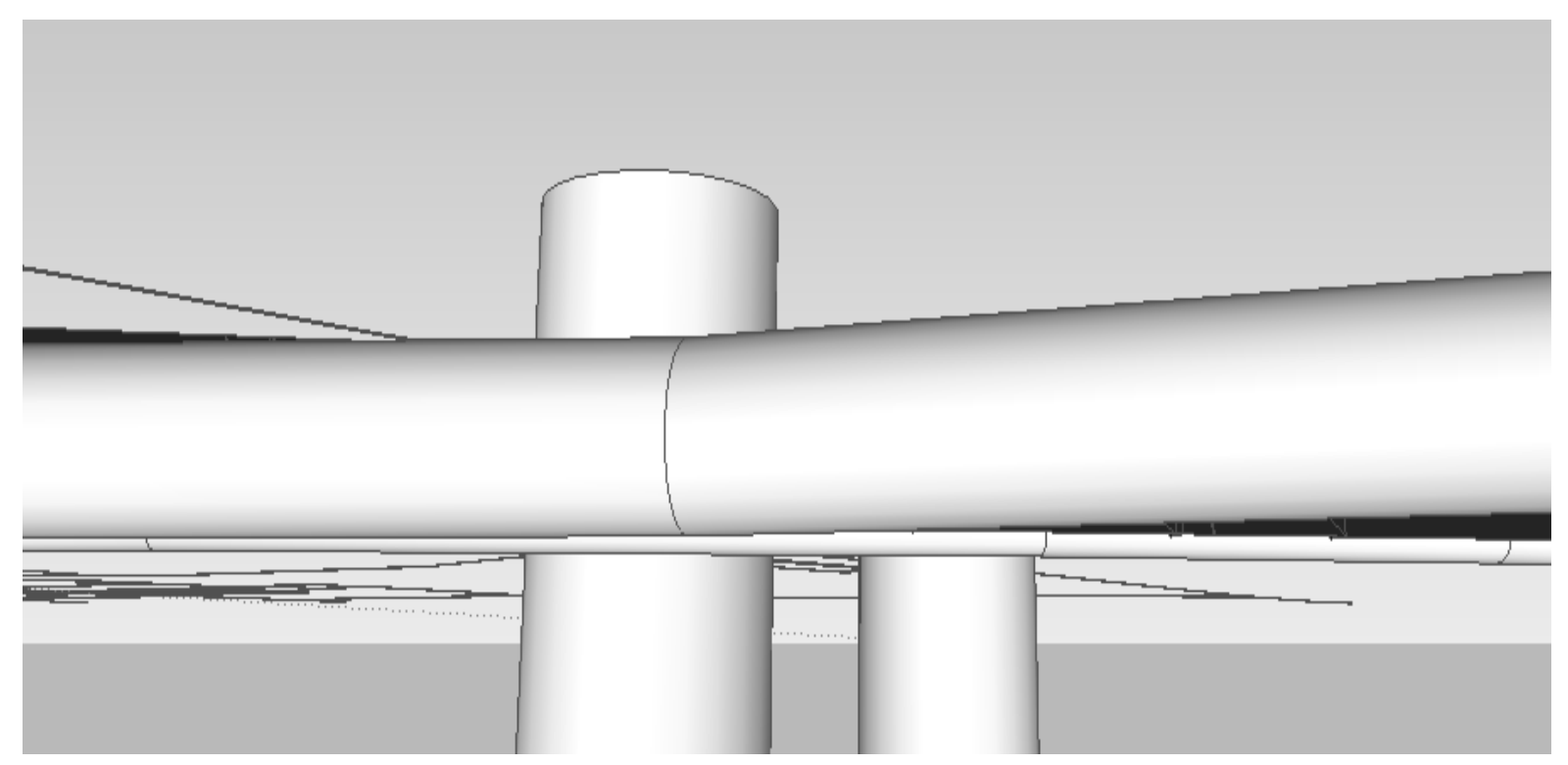

Figure 5. The pipes in 3D format 


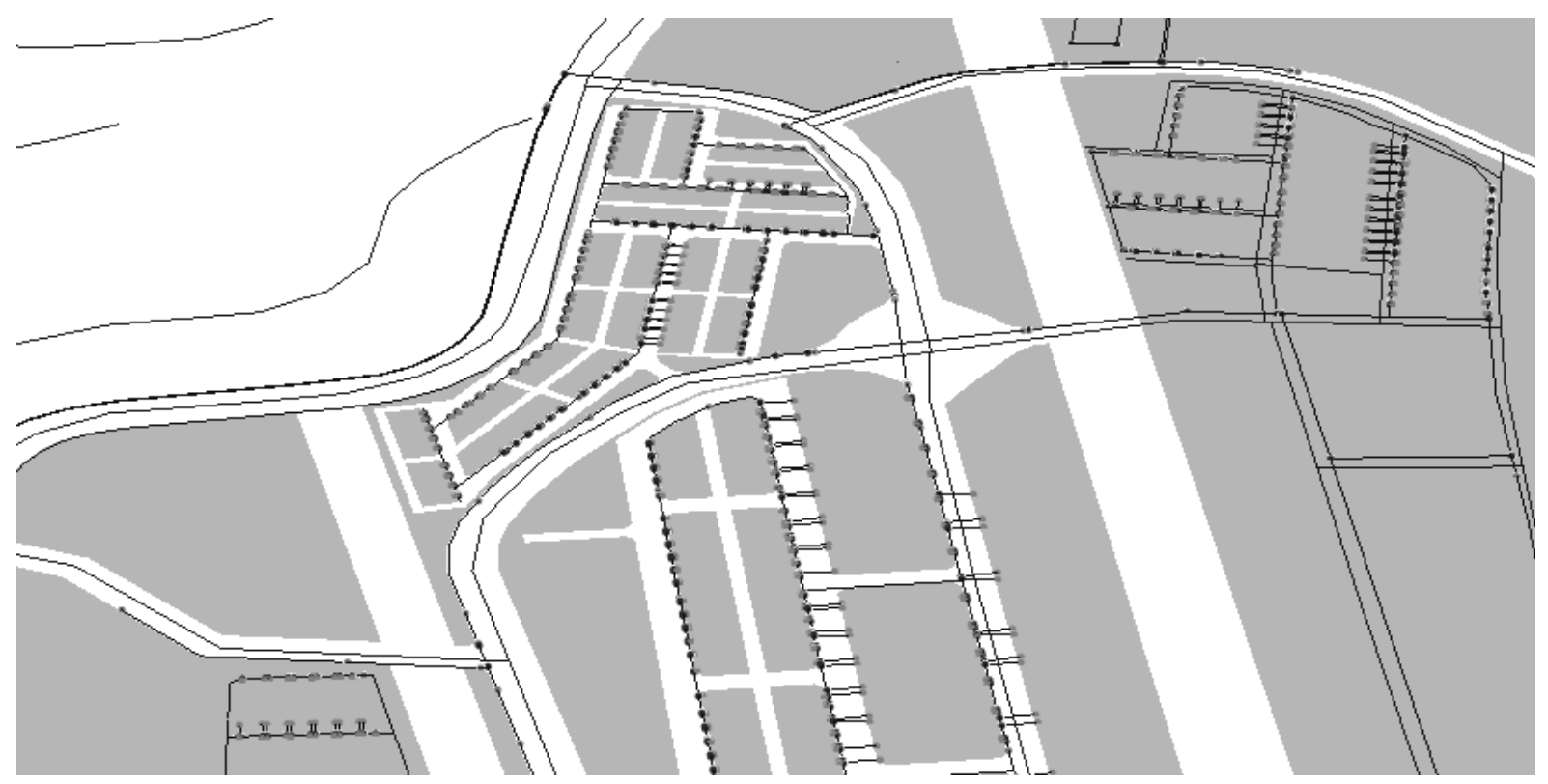

Figure 6. The pipes in 2D format

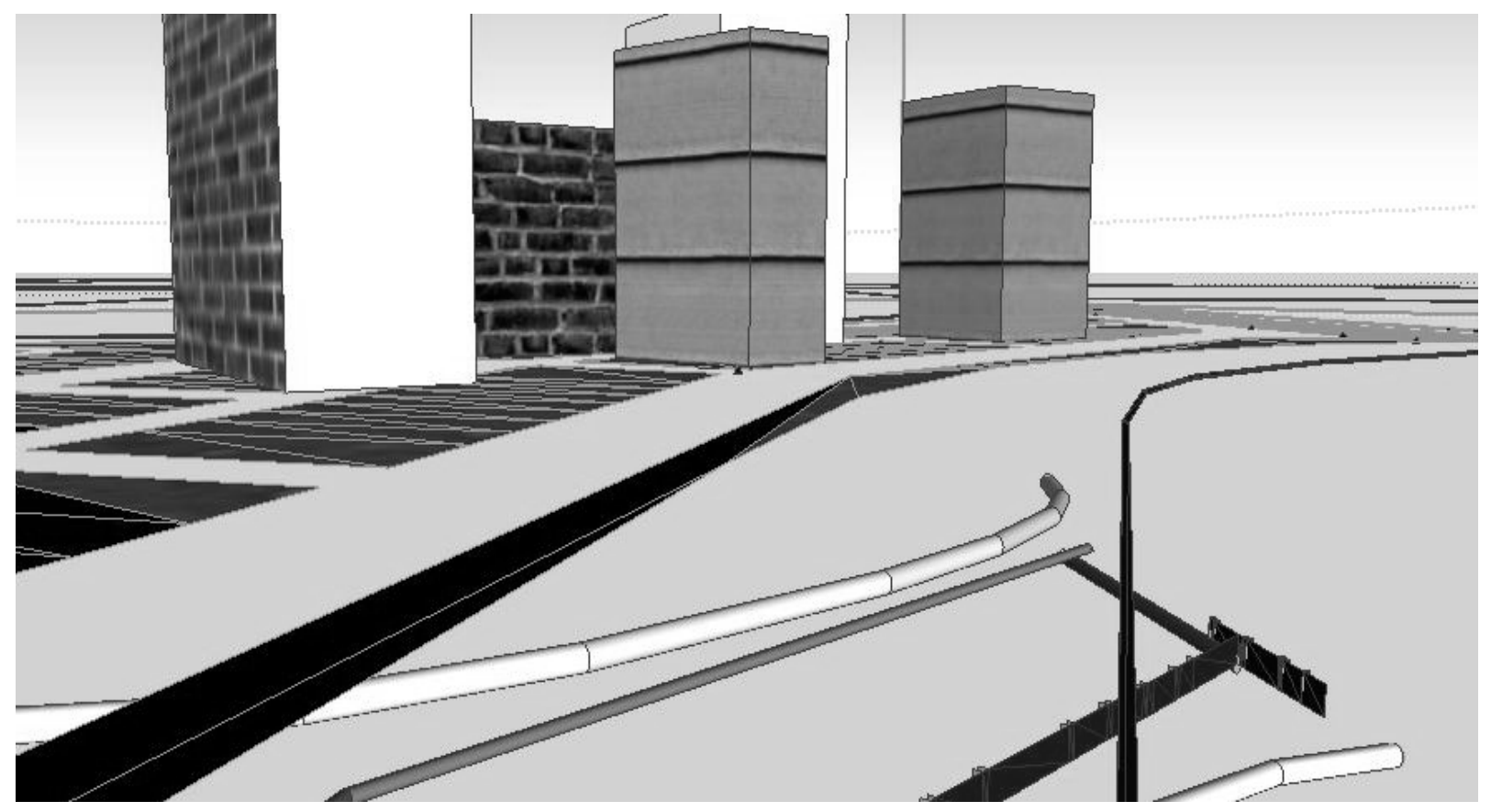

Figure 7. Features modelled in Sketch-up 